\title{
A family of freely slice good boundary links
}

\author{
Jae Choon $\mathrm{Cha}^{1,2} \cdot$ Min Hoon $\mathrm{Kim}^{2} \cdot$ Mark Powell $^{3}$ \\ Received: 30 November 2018 / Revised: 20 July 2019 / Published online: 17 September 2019 \\ (c) The Author(s) 2019
}

\begin{abstract}
We show that every good boundary link with a pair of derivative links on a Seifert surface satisfying a homotopically trivial plus assumption is freely slice. This subsumes all previously known methods for freely slicing good boundary links with two or more components, and provides new freely slice links.
\end{abstract}

Mathematics Subject Classification 57M25 $\cdot 57 \mathrm{~N} 13 \cdot 57 \mathrm{~N} 70$

\section{Introduction}

The still open topological surgery conjecture for 4-manifolds is equivalent to the statement that all good boundary links are freely slice [7, p. 243]. A good boundary link is an $m$-component link $L$ that admits a homomorphism $\phi: \pi_{1}\left(S^{3} \backslash L\right) \rightarrow F$ sending the meridians to the $m$ generators of the free group $F$ of rank $m$, such that the kernel of $\phi$ is perfect, or equivalently such that $H_{1}\left(S^{3} \backslash L ; \mathbb{Z} F\right)=0$. A Whitehead double of a link with vanishing linking numbers is a special case. We say that a link $L$ is freely slice if $L$ bounds slicing discs in $D^{4}$ whose complement has free fundamental group generated by the meridians of $L$.

The goal of this paper is to describe a family of freely slice good boundary links. Previous work, including [8,10-13], has often focussed on Whitehead doubles of links.

Communicated by Thomas Schick.

$凶 \quad$ Mark Powell

mark.a.powell@durham.ac.uk

Jae Choon Cha

jccha@postech.ac.kr

Min Hoon Kim

kminhoon@kias.re.kr

1 Department of Mathematics, POSTECH, Pohang, Gyeongbuk 37673, Republic of Korea

2 School of Mathematics, Korea Institute for Advanced Study, Seoul 02455, Republic of Korea

3 Department of Mathematical Sciences, Durham University, Durham, UK 
A complete list with a detailed discussion can be found in Sect. 6. We expand these results to a much larger class of good boundary links. After stating our main theorem, we will explain the terminology used therein.

Theorem A Suppose that L is a good boundary link that has a Seifert surface admitting a homotopically trivial ${ }^{+}$good basis. Then $L$ is freely slice.

We need to introduce the notion of a good basis on a boundary link Seifert surface, and what it means for a good basis to be homotopically trivial ${ }^{+}$. Consider a collection $V$ of disjoint Seifert surfaces for a boundary link $L$. We call such a collection a boundary link Seifert surface. The Seifert form $\theta: H_{1}(V ; \mathbb{Z}) \times H_{1}(V ; \mathbb{Z}) \rightarrow \mathbb{Z}$ is defined via linking numbers of curves on $V$ and push-offs of such curves into $S^{3} \backslash V$, in direct analogy with the knot case. Choosing a basis for $H_{1}(V ; \mathbb{Z})$ gives rise to a Seifert matrix representing $\theta$. We give a detailed description in Sect. 2.1.

Definition 1.1 A good basis is a collection $\left\{a_{i}, b_{i}\right\}$ of simple closed curves on $V$, representing a basis for $H_{1}(V ; \mathbb{Z})$, whose geometric intersections are $a_{i} \cdot a_{j}=0$, $b_{i} \cdot b_{j}=0, a_{i} \cdot b_{j}=\delta_{i j}$, and such that the Seifert matrix of $V$ with respect to the basis is reducible by a sequence of elementary $S$-reductions to the null matrix. That is, after reordering if necessary, the Seifert matrix with respect to $\left\{a_{1}, b_{1}, a_{2}, b_{2}, \ldots\right\}$ is of the form

$$
\left[\begin{array}{cc:cc:c:cc}
0 & \varepsilon_{1} & 0 & * & \ldots & 0 & * \\
1-\varepsilon_{1} & 0 & 0 & * & & 0 & * \\
\hdashline 0 & 0 & 0 & \varepsilon_{2} & \ldots & 0 & * \\
* & * & 1-\varepsilon_{2} & 0 & \cdots & 0 & * \\
\hdashline \vdots & & \vdots & & \ddots & \vdots \\
\hdashline 0 & 0 & 0 & 0 & \ldots & 0 & \varepsilon_{r} \\
* & * & * & * & \cdots & 1-\varepsilon_{r} & 0
\end{array}\right]
$$

where $\varepsilon_{i}=0$ or 1 for each $i$, and each $*$ designates an arbitrary integer entry.

Note that (1.1) contains no information about the connected component of $V$ on which the pair $\left(a_{i}, b_{i}\right)$ lies. We prove the following fact in Sect. 2.

Corollary 2.2 A boundary link is good if and only if it has a boundary link Seifert surface admitting a good basis.

Recall [19] that an m-component link is said to be homotopically trivial if it is homotopic to the trivial link through immersions of $m$ copies of the circle into $S^{3}$ with disjoint images. The strongest result in the literature on freely slicing good boundary links, due to Freedman and Teichner [13], states that a Whitehead double of a homotopically trivial $^{+}$link is freely slice. Here a link $J$ is said to be homotopically trivial ${ }^{+}$ if for each component $J_{i}$ of $J$, the link $J \cup J_{i}^{+}$is homotopically trivial, where $J_{i}^{+}$is a zero linking parallel copy of $J_{i}$.

Our notion of a homotopically trivial ${ }^{+}$good basis extends the Freedman-Teichner definition for links to a more general context. Let $\left\{a_{i}, b_{i}\right\}$ be a good basis on a boundary link Seifert surface $V$. Inspecting the diagonal blocks of (1.1), it follows that $a_{i}$ has 
Fig. 1 Standard good basis for a Whitehead double

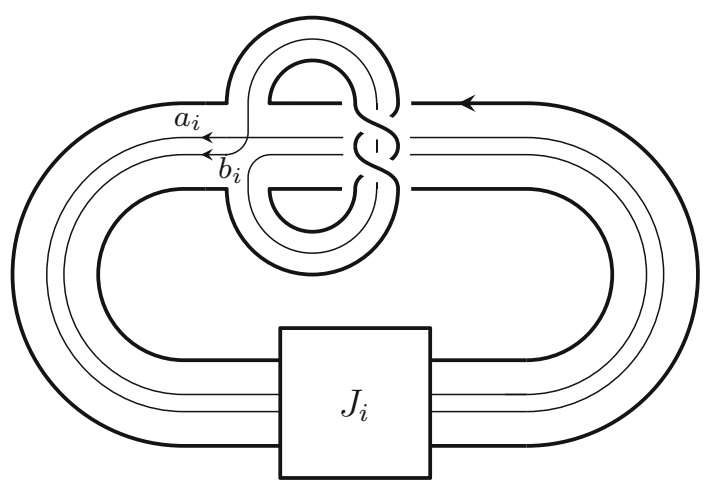

trivial linking number with exactly one of the two translates of $b_{i}$ in the positive and negative normal directions of $V$. Call this push-off $b_{i}^{\prime}$ and let $K:=\bigsqcup b_{i}^{\prime}$.

Definition 1.2 We call the good basis $\left\{a_{i}, b_{i}\right\}$ homotopically trivial ${ }^{+}$if both of the links $K \sqcup a_{j}$ and $K \sqcup b_{j}$ are homotopically trivial for every $j$.

Observe that if $\left\{a_{i}, b_{i}\right\}$ is a homotopically trivial ${ }^{+}$good basis then each of the sets of curves $\left\{a_{i}\right\}$ and $\left\{b_{i}\right\}$ is a derivative of $L$, that is a collection of mutually disjoint curves on a Seifert surface for $L$ whose linking and self-linking numbers all vanish. In fact all the entries of (1.1) indicated by a $*$ are 0 for a homotopically trivial ${ }^{+}$good basis. Boundary links admitting this kind of Seifert matrix were termed generalised doubles by Freedman and Krushkal in [5, Definition 5.9].

To understand the connection between the two notions of homotopically trivial ${ }^{+}$, consider $\mathrm{Wh}(J)$, an untwisted Whitehead double of the link $J$ with arbitrary clasp signs. Recall that $\mathrm{Wh}(J)$ bounds a standard boundary link Seifert surface with each connected component genus one. Figure 1 illustrates the case of a positive clasp. It is straightforward to verify the following.

(i) If $J$ has vanishing pairwise linking numbers, so that $\mathrm{Wh}(J)$ is a good boundary link, then the curves $a_{i}$ and $b_{i}$ shown in Fig. 1 form a good basis.

(ii) The good basis $\left\{a_{i}, b_{i}\right\}$ is homotopically trivial ${ }^{+}$if and only if the link $J$ is homotopically trivial ${ }^{+}$in the sense of Freedman-Teichner. We provide the details in Lemma 6.1.

Thus the fact that Whitehead doubles of homotopically trivial ${ }^{+}$links are slice is a special case of Theorem A. Moreover, Theorem A provides new freely slice links, not previously known to be slice. In particular, in Sect. 6.1, we provide examples not arising as a Whitehead double.

We close the introduction by noting that it is logically conceivable that Theorem A applies to freely slice Whitehead doubles of non-homotopically trivial $^{+}$links. We discuss further related questions in Sect. 7. 


\section{Seifert matrices of boundary links}

In this section we present certain facts on Seifert matrices of boundary links and $S$ equivalence, which may be known to the experts. We could not find these facts in the literature, so we provide the proofs. The main goal of the section is to prove the following proposition.

Proposition 2.1 A boundary link $L$ is good if and only if there is a boundary link Seifert surface for $L$ such that the associated Seifert matrix can be changed to a null matrix by $S$-reductions only.

We will give the definitions of the terms used in Proposition 2.1 in Sect. 2.1 below. The following appeared as a proposition in the introduction.

Corollary 2.2 A boundary link is good if and only if it has a boundary link Seifert surface admitting a good basis.

We prove the corollary assuming Proposition 2.1 and Theorem 2.11 below, which states that a boundary link $L$ is good if and only if some (and therefore every) Seifert matrix for $L$ is $S$-equivalent to the null matrix.

Proof Let $L$ be a good boundary link and let $V=\bigsqcup V_{i}$ be a boundary link Seifert surface for $L$ as given by Proposition 2.1, where $V_{i}$ is a Seifert surface for the $i$ th component. We may and shall assume that each $V_{i}$ is connected. Fix a basis for $H_{1}\left(V_{i} ; \mathbb{Z}\right)$, for each $i$, such that the corresponding Seifert matrix $A$ can be changed to a null matrix by $S$-reductions. The basis is symplectic with respect to the intersection form, since $A-A^{T}$, which represents the intersection form by Definition 2.3 and the subsequent paragraph, is a diagonal sum of matrices of the form $\pm\left[\begin{array}{cc}0 & 1 \\ -1 & 0\end{array}\right]$, by Definition 2.5 (ii) and (iii) below. Since every symplectic automorphism of $H_{1}\left(V_{i} ; \mathbb{Z}\right)$ is realised by a homeomorphism of $V_{i}$ (e.g., see [6, Theorem 6.4]), this basis can be represented by simple closed curves whose geometric intersections match their algebraic intersection numbers. By definition, these curves form a good basis.

For the converse, suppose that a boundary link $L$ has a Seifert surface admitting a good basis. Then $L$ has a Seifert matrix which is $S$-equivalent to a null matrix, so $L$ is good by Theorem 2.11 .

\subsection{Seifert matrices and S-equivalence}

We begin with the basic definitions. We always assume that an $m$-component boundary link $L=L_{1} \sqcup \cdots \sqcup L_{m}$ is endowed with a choice of epimorphism $\phi: \pi_{1}\left(S^{3} \backslash L\right) \rightarrow F$ onto a free group $F$ of rank $m$ that sends the $i$ th meridian to the $i$ th free generator. This is equivalent to assuming, via the Pontryagin-Thom construction, that $L$ comes with a choice of $m$ disjoint Seifert surfaces $V_{i}$ with $\partial V_{i}=L_{i}$. A boundary link with such a choice is often called an $F$-link.

The standard definition of a Seifert matrix of a knot generalises to the case of boundary links, as follows. 
Definition 2.3 Define a bilinear pairing $H_{1}\left(V_{i} ; \mathbb{Z}\right) \times H_{1}\left(V_{j} ; \mathbb{Z}\right) \rightarrow \mathbb{Z}$ by $([x],[y]) \mapsto$ $\operatorname{lk}\left(x^{+}, y\right)$ for 1-cycles $x$ and $y$, where $x^{+}$denotes a push-off of $x$ along the positive normal direction of $V_{i}$. Choosing a basis of $H_{1}\left(V_{i} ; \mathbb{Z}\right)$ for each $i$ determines a matrix $A_{i j}$. The block matrix $A=\left[A_{i j}\right]_{1 \leq i, j \leq m}$ comprising $m^{2}$ submatrices $A_{i j}$ is called a Seifert matrix for $L$.

We remark that $A_{i i}-A_{i i}^{T}$ represents the intersection form of $V_{i}$ and consequently is unimodular by Poincaré-Lefschetz duality on $V_{i}$ and the isomorphism $H_{1}\left(V_{i} ; \mathbb{Z}\right) \cong$ $H_{1}\left(V_{i}, \partial V_{i} ; \mathbb{Z}\right)$. Also, $A_{i j}=A_{j i}^{T}$ for $i \neq j$ by symmetry of the linking number.

The notion of $S$-equivalence for Seifert matrices of knots generalises to the case of boundary links [18]. The next two definitions are algebraic.

Definition 2.4 We say that a square integral matrix $A$ is an $m$-component boundary link Seifert matrix if it has a decomposition into $m^{2}$ blocks $A_{i j}(i, j=1, \ldots, m)$ with $A_{i i}$ a square matrix for each $i$, such that $\operatorname{det}\left(A_{i i}-A_{i i}^{T}\right)= \pm 1$ and $A_{i j}=A_{j i}^{T}$ for $i \neq j$.

Definition 2.5 Suppose $A=\left[A_{i j}\right]$ and $B=\left[B_{i j}\right]$ are $n$-component boundary link Seifert matrices.

(i) We say that $A$ and $B$ are congruent if there are square unimodular matrices $P_{1}, \ldots, P_{m}$ such that $P_{i}^{T} A_{i j} P_{j}=B_{i j}$ for all $i, j$. That is, for

$$
P=\left[\begin{array}{lll}
P_{1} & & \\
& \ddots & \\
& & P_{m}
\end{array}\right],
$$

the block matrix product $P^{T} A P$ equals $B$.

(ii) We say that a square integral matrix $B$ is an $S$-enlargement of $A$ if $B$ is of the form

$$
B=\left[\begin{array}{c|c|ccc|c|c}
\ddots & \vdots & & \vdots & \vdots & \cdot \\
\hline \cdots & A_{k-1, k-1} & 0 & x_{k-1}^{T} & A_{k-1, k} & A_{k-1, k+1} & \cdots \\
\hline & 0 & 0 & \varepsilon & 0 & 0 & \\
\cdots & x_{k-1} & \varepsilon^{\prime} & 0 & x_{k} & x_{k+1} & \cdots \\
& A_{k, k-1} & 0 & x_{k}^{T} & A_{k k} & A_{k, k+1} & \\
\hline \cdots & A_{k+1, k-1} & 0 & x_{k+1}^{T} & A_{k+1, k} & A_{k+1, k+1} & \cdots \\
\hline \cdot & \vdots & & \vdots & \vdots & \ddots
\end{array}\right]
$$


for some $k$, where $\left(\varepsilon, \varepsilon^{\prime}\right)=(1,0)$ or $(0,1)$ and $x_{i}$ designates an arbitrary row vector of the appropriate dimension for $i=1, \ldots, m$. That is, $B_{i j}$ is equal to

$$
\begin{aligned}
& {\left[\begin{array}{ccc}
0 & \varepsilon^{\prime} & 0 \\
\varepsilon & 0 & x_{k} \\
0 & x_{k}^{T} & A_{k k}
\end{array}\right] \text { for } i=k, j=k, \quad\left[\begin{array}{c}
0 \\
x_{j} \\
A_{k j}
\end{array}\right] \text { for } i=k, j \neq k,} \\
& {\left[\begin{array}{lll}
0 & x_{i}^{T} & A_{i k}
\end{array}\right] \text { for } i \neq k, j=k, \quad A_{i j} \quad \text { for } i \neq k, j \neq k .}
\end{aligned}
$$

(iii) We say that $B$ is an $S$-reduction of $A$ if $A$ is an $S$-enlargement of $B$.

(iv) Finally, we say that $A$ and $B$ are $S$-equivalent if one can be obtained from another by a finite sequence of congruences, $S$-enlargements, and $S$-reductions.

Proposition 2.6 ([18, Theorem 1]) Any two boundary link Seifert matrices of the same boundary link are $S$-equivalent.

When $B$ is an $S$-enlargement of $A$, we write $A \nearrow B$, or $B \searrow A$. When $A$ and $B$ are congruent, we write $A \sim B$. The following lemma tells us that for any sequence of the moves described in the definition of $S$-equivalence, a local minimum of the size of a Seifert matrix can be replaced by a local maximum.

Lemma 2.7 If $A$ and $B$ are Seifert matrices such that $A \searrow C \sim C^{\prime} \nearrow B$ for some $C$, $C^{\prime}$, then there are Seifert matrices $D, D^{\prime}$ such that $A \nearrow D \sim D^{\prime} \searrow B$.

Proof We start with a proof in the two component case, that is $m=2$. We consider the case that the reduction and enlargement happen to the same block, since the other cases are straightforward. Let $C=\left[C_{i j}\right]$ and $C^{\prime}=P^{T} C P$ for $P=\operatorname{diag}\left(P_{1}, P_{2}\right)$. Then

$$
A=\left[\begin{array}{ccc|c}
0 & \varepsilon & 0 & 0 \\
\varepsilon^{\prime} & 0 & x_{1} & x_{2} \\
0 & x_{1}^{T} & C_{11} & C_{12} \\
\hline 0 & x_{2}^{2} & C_{21} & C_{22}
\end{array}\right], \quad B=\left[\begin{array}{ccc|c}
0 & \delta & 0 & 0 \\
\delta^{\prime} & 0 & y_{1} & y_{2} \\
0 & y_{1}^{T} & P_{1}^{T} C_{11} P_{1} & P_{1}^{T} C_{12} P_{2} \\
\hline 0 & y_{2}^{2} & P_{2}^{T} C_{21} P_{1} & P_{2}^{T} C_{22} P_{2}
\end{array}\right]
$$

for some $x_{i}, y_{i}, \varepsilon, \varepsilon^{\prime}, \delta$, and $\delta^{\prime}$. Now we have $A \nearrow D \sim Q^{T} D Q \searrow B$ for

$$
D=\left[\begin{array}{ccccc|c}
0 & \delta & 0 & 0 & 0 & 0 \\
\delta^{\prime} & 0 & 0 & 0 & y_{1} P_{1}^{-1} & y_{2} P_{2}^{-1} \\
0 & 0 & 0 & \varepsilon & 0 & 0 \\
0 & 0 & \varepsilon^{\prime} & 0 & x_{1} & x_{2} \\
0\left(P_{1}^{-1}\right)^{T} & y_{1}^{T} & 0 & x_{1}^{T} & C_{11} & C_{12} \\
\hline 0\left(P_{2}^{-1}\right)^{T} y_{2}^{T} & 0 & x_{2}^{T} & C_{21} & C_{22}
\end{array}\right] \text { and }
$$




$$
Q=\left[\begin{array}{lllll|l}
1 & & & & & \\
& 1 & & & & \\
& & 1 & & & \\
& & & 1 & & \\
& & & & P_{1} & \\
& & & & \\
\hline & & & & P_{2}
\end{array}\right]
$$

The general case is verified analogously, since as mentioned above the only interesting case is that the reduction and the enlargement happen to one block. Without loss of generality we may assume it is the top left block. The matrices $D$ and $Q$ above extend in a natural way.

Repeatedly apply Lemma 2.7 to remove all local minima of the size of $A$, so that there is at most one maximum. We record the outcome of this procedure.

Corollary 2.8 Suppose $A$ and $B$ are S-equivalent. Then $A$ can be changed to $B$ by a sequence of congruences, $S$-enlargements, and $S$-reductions in which no $S$ enlargement appears after any $S$-reduction.

Here is another elementary observation that enables us to rearrange the order of operations applied to a Seifert matrix.

Lemma 2.9 If $B$ results from A after $S$-reductions and congruences, applied in any order, then $B$ can be obtained from A by applying a single congruence first and then applying $S$-reductions only.

Proof It suffices to consider the case of $A \searrow P^{T} B P \sim B$. Once again, for brevity, we will consider the two component case only, and in addition just like in proof of the preceding lemma we assume without loss of generality that the $S$-reduction acts on the first block of the Seifert matrix. The same argument applies to the general case of more than two components, with the natural extension of the matrices. Write $B=\left[B_{i j}\right]$ and $P=\operatorname{diag}\left(P_{1}, P_{2}\right)$. We have

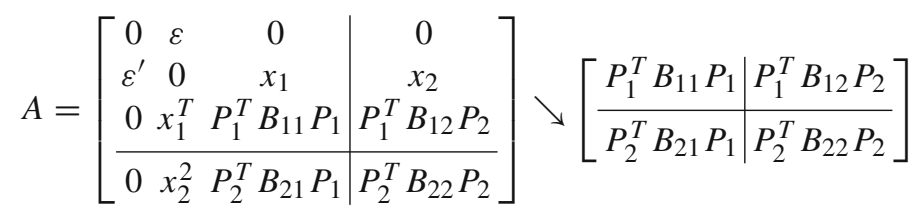

and this equals $P^{T} B P \sim B$. Let $Q=\operatorname{diag}\left(1,1, P_{1}^{-1}, P_{2}^{-1}\right)$. Then

$$
A \sim Q^{T} A Q=\left[\begin{array}{ccc|c}
0 & \varepsilon & 0 & 0 \\
\varepsilon^{\prime} & 0 & x_{1} P_{1}^{-1} & x_{2} P_{2}^{-1} \\
0\left(P_{1}^{-1}\right)^{T} x_{1}^{T} & B_{11} & B_{12} \\
\hline 0\left(P_{2}^{-1}\right)^{T} x_{2}^{2} & B_{21} & B_{22}
\end{array}\right] \searrow B
$$

gives a congruence followed by an $S$-reduction instead. 
The above observations enable us to relate two Seifert matrices by a sequence of operations that are monotone with respect to the matrix size.

Lemma 2.10 Suppose $L$ and $L^{\prime}$ are boundary links in $S^{3}$ with $S$-equivalent Seifert matrices. Then for any given Seifert matrix $A^{\prime}$ of $L^{\prime}$, the other link $L$ has a Seifert matrix that can be changed to $A^{\prime}$ by a sequence of $S$-reductions.

Proof Let $A$ be a Seifert matrix of $L$. By hypothesis, $A$ is $S$-equivalent to $A^{\prime}$. By Corollary 2.8, $A$ can be changed, by congruences and $S$-enlargements, to some $B$ that can then itself be changed to $A^{\prime}$ by a sequence of congruences and $S$-reductions. Since an $S$-enlargement of a Seifert matrix is realised by 1 -surgery on any given Seifert surface with respect to which the Seifert matrix is defined, it follows that there is a Seifert surface $V$ for $L$ that has $B$ as its Seifert matrix. By Lemma 2.9, a basis change of the first homology transforms $B$ to a Seifert matrix for $L$ defined on the same $V$ that can be changed to $A^{\prime}$ by $S$-reductions only.

\subsection{S-equivalence and good boundary links}

Recall that a boundary link $L$ is said to be a good boundary link if the given epimorphism $\phi: \pi_{1}\left(S^{3} \backslash L\right) \rightarrow F$ has a perfect kernel, or equivalently if $H_{1}\left(S^{3} \backslash L ; \mathbb{Z} F\right)=$ 0 . Since the Blanchfield form on $H_{1}\left(S^{3} \backslash L ; \mathbb{Z} F\right)$ is nondegenerate [14, Section 2.7], we see that $H_{1}\left(S^{3} \backslash L ; \mathbb{Z} F\right)$ has no $\mathbb{Z}$-torsion. It follows that $L$ is good if and only if $H_{1}\left(S^{3} \backslash L ; \mathbb{Q} F\right)=0$.

Theorem 2.11 ([4,18]) A boundary link $L$ in $S^{3}$ is good if and only if some (and thence every) Seifert matrix for $L$ is $S$-equivalent to the null matrix.

In the following proof, we combine results on high dimensional boundary links due to $[4,15,17,18,22]$.

Proof Let $A$ be a Seifert matrix for $L$. Every Seifert matrix can be realised by a simple boundary link in an arbitrary odd dimension (see Theorem 2 of [18], Theorem 3.4 of [15]). Thus there is a simple boundary link $L^{\prime}$ of codimension two in $S^{4 k-1}$ with $k>1$ that has the same Seifert matrix $A$. Here a boundary link in $S^{4 k-1}$ is said to be simple if the components have disjoint and $(2 k-2)$-connected Seifert surfaces.

By [17,22], the module $H_{2 k-1}\left(S^{4 k-1} \backslash L^{\prime} ; \mathbb{Q} F\right)$ has a presentation determined by the Seifert matrix. It follows that the modules $H_{2 k-1}\left(S^{4 k-1} \backslash L^{\prime} ; \mathbb{Q} F\right)$ and $H_{1}\left(S^{3} \backslash L ; \mathbb{Q} F\right)$ are isomorphic.

By [18, Theorem 3], a high dimensional simple boundary link is a trivial link if and only if it has a Seifert matrix $S$-equivalent to a null matrix. Also, by [4, Theorem 6.7], $L^{\prime}$ is a trivial link if and only if $H_{2 k-1}\left(S^{4 k-1} \backslash L^{\prime} ; \mathbb{Q} F\right)=0$. It follows that $H_{1}\left(S^{3} \backslash L ; \mathbb{Q} F\right)=0$ if and only if the Seifert matrix $A$ is $S$-equivalent to the null matrix.

Proof of Proposition 2.1 Combine Theorem 2.11 with Lemma 2.10, applied with $L^{\prime}$ the unlink and with discs as Seifert surfaces, so $A^{\prime}$ is the null matrix. This shows that there is a Seifert surface $V=\bigsqcup V_{i}$ for $L$ such that the associated Seifert matrix can be changed to a null matrix by $S$-reductions only. 


\section{Homotopically trivial ${ }^{+}$pairs}

In this section, we generalise the notion of homotopically trivial ${ }^{+}$links, defined in [13, Section 3], to pairs consisting of a link and a sublink. Recall that an $m$-component link $J$ in $S^{3}$ is homotopically trivial if there is a homotopy of $J$ to the $m$-component unlink in $S^{3}$ through immersions of $m$ circles with disjoint images.

Definition 3.1 For a sublink $K$ of a link $J$ in $S^{3}$, we say that the pair $(J, K)$ is homotopically trivial ${ }^{+}$if $K \cup J_{i}^{+}$is homotopically trivial for every component $J_{i}$ of $J$, where $J_{i}^{+}$is a zero linking parallel copy of $J_{i}$.

Remark 3.2 (1) By inspection of the definition, a good basis $\left\{a_{i}, b_{i}\right\}$ on a boundary link Seifert surface $V$ is homotopically trivial ${ }^{+}$if and only if the pair $\left(\bigsqcup\left(a_{i} \cup b_{i}^{\prime}\right), \bigsqcup b_{i}^{\prime}\right)$ is homotopically trivial ${ }^{+}$, where $b_{i}^{\prime}$ is a normal translate of $b_{i} \subset V$ satisfying $\operatorname{lk}\left(a_{i}, b_{i}^{\prime}\right)=0$.

(2) The pair $(J, J)$ is homotopically trivial ${ }^{+}$if and only if $J$ is homotopically trivial ${ }^{+}$ in the sense of [13]. More generally, suppose that $J$ is a ramification of a link $K$, that is $J$ consists of $K$ together with zero linking parallel copies of a (not necessarily proper) sublink of $K$. Then $(J, K)$ is homotopically trivial ${ }^{+}$if and only if $K$ is homotopically trivial ${ }^{+}$.

The next lemma extends [13, Lemma 3.2] to homotopically trivial ${ }^{+}$link pairs.

Lemma 3.3 Let $(J, K)$ be a homotopically trivial ${ }^{+}$pair. Then there are two collections of immersed discs $\left\{\Delta_{i}\right\}$ and $\left\{\Delta_{k}^{+}\right\}$in $D^{4}$ satisfying that $\partial \Delta_{i}=K_{i}, \partial \Delta_{k}^{+}=J_{k}^{+}$, $\Delta_{i} \cap \Delta_{k}=\emptyset$ for all $i \neq k$ and $\Delta_{i} \cap \Delta_{k}^{+}=\emptyset$ for all $i$ and $k$.

We will give two proofs of Lemma 3.3. The first is essentially identical to the proof of [13, Lemma 3.2], which uses the Milnor group introduced in [19]. The second proof gives an elementary geometric construction of the desired discs without using algebra. We believe that this is a useful addition to the literature. It uses a homotopy reversing method that appeared in [16, Section 3.4].

Proof using the Milnor group Let $m$ be the number of components of $K$. Choose disjointly immersed discs $\Delta_{i}$ in $D^{4}$ bounded by $K_{i}$ for $i=1, \ldots, m$, using that $K$ is homotopically trivial. Let $\pi=\pi_{1}\left(S^{3} \backslash \bigsqcup K_{i}\right), G=\pi_{1}\left(D^{4} \backslash \bigsqcup \Delta_{i}\right)$ and $F$ be the free group on $x_{1}, \ldots, x_{m}$. Let $F \rightarrow \pi \rightarrow G$ be the map sending $x_{i}$ to a meridian of $K_{i}$, and denote the image of $x_{i}$ in $\pi$ and $G$ by the same symbol $x_{i}$. For $\Gamma=F, \pi$ and $G$, the Milnor group $M \Gamma$ is defined to be the quotient of $\Gamma$ modulo relators $\left[x_{i}^{g}, x_{i}\right]$, $i=1, \ldots, m, g \in \Gamma$ [19]. The group $M \Gamma$ is finitely generated and nilpotent [19], and consequently finitely presented. For $\Gamma=G$, since a relator $\left[x_{i}^{g}, x_{i}\right]$ is realised by a finger move on $\Delta_{i}$, we may assume that $M G=G$ by applying finitely many finger moves to the discs. Moreover, the following facts are known: $M \pi \cong M F$ since $K$ is homotopically trivial [19, Section 5], and $K \cup J_{k}^{+}$is homotopically trivial if and only if $\left[J_{k}^{+}\right]$is trivial in $M \pi\left[19\right.$, Section 5]. Also, $M F \cong M G$ since $\bigsqcup \Delta_{i}$ is a link null-homotopy [13, Lemma 2.6]. It follows that $\left[J_{k}^{+}\right]$is trivial in $M G=G$ for all $k$. Therefore there exist immersed discs $\Delta_{k}^{+}$(which may intersect each other) in $D^{4} \backslash \bigsqcup \Delta_{i}$ satisfying $\partial \Delta_{k}^{+}=J_{k}^{+}$. 
Constructive geometric proof Let $n$ be the number of components of $J$ and let $m$ be the number of components of $K$. Let $J^{+}$be the union of pushed off parallel copies $J_{k}^{+}$of the components of $J$.

We work by induction on the components of $J^{+}$to construct annuli in $S^{3} \times I$, which will then be capped off by discs to obtain the desired immersed discs, after embedding $S^{3} \times I \subset D^{4}$ as a collar on the boundary. For the induction, $j$ will range from 1 to $n$, corresponding to the indices of the components of $J$. Fix a 3-ball $B$ in $S^{3}$ disjoint from $K \cup J^{+}$. Suppose for the inductive hypothesis that we have constructed a level preserving immersion of $n+m$ annuli $\left\{A_{i}\right\}_{i=1}^{m}$ and $\left\{A_{k}^{+}\right\}_{k=1}^{n}$ in $S^{3} \times I=S^{3} \times[0,1]$ with $A_{i} \cap\left(S^{3} \times\{0\}\right)=K_{i}$ for $i=1, \ldots, m$ and $A_{k}^{+} \cap\left(S^{3} \times\{0\}\right)=J_{k}^{+}$for $k=1, \ldots, n$, such that

(i) $A_{i} \cap A_{\ell}=\emptyset$ for all $i \neq \ell$ with $1 \leq i, \ell \leq m$;

(ii) $A_{i} \cap A_{k}^{+}=\emptyset$ for all $i$ and $k$ with $1 \leq i \leq m$ and $1 \leq k \leq n$;

(iii) $\left(A_{1}^{+} \cup \cdots \cup A_{j-1}^{+}\right) \cap\left(S^{3} \times\{1\}\right)$ is a trivial link contained in $B \times\{1\}$, while $A_{1}, \ldots, A_{m}$ and $A_{j}^{+}, \ldots, A_{n}^{+}$are disjoint from $B \times\{1\}$; and

(iv) $A_{i} \cap\left(S^{3} \times\{1\}\right)=K_{i}$ for $i=1, \ldots, m$ and $A_{k}^{+} \cap\left(S^{3} \times\{1\}\right)=J_{k}^{+}$for $k=j, \ldots, n$.

We seek to extend these annuli in $S^{3} \times[0,4]$ so that we have the same property for $j$ replaced by $j+1$. Then we will rescale the interval $[0,4]$ to $[0,1]=I$ to achieve the inductive step. In the base case that $j=1$, take the annuli to be a product concordance.

In what follows, for brevity, we denote extensions of $A_{i}$ and $A_{k}^{+}$by $A_{i}$ and $A_{k}^{+}$. For $k=1, \ldots, j-1$, extend $A_{k}^{+}$by attaching the product $J_{k}^{+} \times[1,2]$, which lies in $B \times[1,2]$. Use the hypothesis that $K \cup J_{j}^{+}$is homotopically trivial to find disjointly immersed level preserving annuli extending $A_{1}, \ldots, A_{m}$ and $A_{j}^{+}$in $S^{3} \times[1,2]$, ending in a trivial link in $S^{3} \times\{2\}$. We may and shall assume that these annuli are disjoint from $B \times[1,2]$, since a link null-homotopy for $K \cup J_{j}^{+}$in $S^{3}$ can be arranged to avoid $B$. Furthermore, since link homotopy is generated by crossing changes and ambient isotopy, and since crossing changes can be assumed to be performed along arcs disjoint from $J_{j+1}^{+}, \ldots, J_{n}^{+}$, the annuli $A_{j+1}^{+}, \ldots, A_{n}^{+}$extend to disjointly embedded annuli in $S^{3} \times[1,2]$, ending in some link in $S^{3} \times\{2\}$. The annuli $A_{j+1}^{+}, \ldots, A_{n}^{+}$do not meet $B \times[1,2]$ and the above (extensions of) $A_{1}, \ldots, A_{m}, A_{j}^{+}$.

Next, we proceed to extend the annuli in $S^{3} \times[2,3]$. Choose an isotopy, in $S^{3} \times\{2\}$, of the end of $A_{j}^{+}$in $S^{3} \times\{2\}$ into the ball $B$. We may assume that the isotopy does not meet $A_{1}, \ldots, A_{m}$ and $A_{1}^{+}, \ldots, A_{j-1}^{+}$in $S^{3} \times\{2\}$. Use the level preserving embedded annulus corresponding to this isotopy to extend $A_{j}^{+}$in $S^{3} \times[2,3]$. Extend $A_{i}$ and $A_{k}^{+}$, except for $k=j$, into $S^{3} \times[2,3]$ by attaching product annuli. Then $A_{j}^{+}$is disjoint from the annuli $A_{i}$ and $A_{k}^{+}$for $k<j$, while $A_{j}^{+}$may intersect $A_{k}^{+}$for $k>j$. Note that these intersections are permitted in the inductive hypothesis and ultimately in the discs that we aim to construct.

Finally, extend the annuli $A_{i}, A_{k}^{+}$once more, into $S^{3} \times[3,4]$, as follows. For the components inside $B \times\{3\}$, that is the boundary components of the annuli $A_{1}^{+}, \ldots, A_{j}^{+}$ in $S^{3} \times\{3\}$, extend them by attaching product annuli. For the remaining components, 
perform the reverse of the homotopies applied thus far. That is, for $A$ one of the annuli $A_{1}, \ldots, A_{m}$ or $A_{k}^{+}$with $k>j$, which lies in $S^{3} \times[0,3]$ for now, extend $A$ by attaching the image of $A \cap\left(S^{3} \times[1,3]\right)$ under the orientation reversing diffeomorphism $S^{3} \times[1,3] \rightarrow S^{3} \times[3,4],(x, t) \mapsto\left(x, \frac{9}{2}-\frac{t}{2}\right)$. We have arranged that the boundary components of the annuli $A_{j+1}^{+}, \ldots, A_{n}^{+}$and $A_{1}, \ldots, A_{m}$ that lie in $S^{3} \times\{4\}$ coincide with $J_{j+1}^{+}, \ldots, J_{n}^{+}$and $K_{1}, \ldots, K_{m}$, so that after rescaling $[0,4]$ to $[0,1]$, condition (iv) is satisfied, with $j$ replaced by $j+1$. It is clear that no new intersections between any pair of the annuli $A_{j+1}^{+}, \ldots, A_{n}^{+}$and $A_{1}, \ldots, A_{m}$ are introduced in $S^{3} \times[3,4]$, unless that pair already intersected in $S^{3} \times[1,3]$. For $A_{1}^{+}, \ldots, A_{j}^{+}$, observe that their extensions in $S^{3} \times[3,4]$ are contained in $B \times[3,4]$, while other annuli are disjoint from $B \times[1,4]$, by the previous two steps. So, there are no new intersections involving $A_{1}^{+}, \ldots, A_{j}^{+}$. This shows that conditions (i) and (ii) hold for the annuli we have constructed. Finally, we have moved the component $J_{j}^{+}$into the ball $B$, and so condition (iii) holds with $j$ replaced by $j+1$. This completes the proof of the inductive step.

After the induction has been run for all $j$, we obtain a collection of immersed annuli in $S^{3} \times I$ satisfying the conditions above for $j=n+1$. Cap off the annuli $\left\{A_{k}^{+}\right\}_{k=1}^{n}$ with discs inside $B \times\{1\}$. Then cap $S^{3} \times I$ off with a 4-ball, to obtain $S^{3} \times I \cup D^{4} \cong D^{4}$. It remains to cap off the annuli $\left\{A_{i}\right\}_{i=1}^{m}$. The intersection of these annuli with $S^{3} \times\{1\}$ form the link $K$. Since $K$ is homotopically trivial, it can be capped off with disjointly immersed discs in the capping-off $D^{4}$. Let $\Delta_{i}$ and $\Delta_{k}^{+}$be the discs obtained from capping off $A_{i}$ and $A_{k}^{+}$respectively. The collections of discs $\left\{\Delta_{i}\right\}$ and $\left\{\Delta_{k}^{+}\right\}$satisfy the desired disjointness properties.

\section{Freely slicing a boundary link by finding a slice disc exterior}

Let $M_{L}$ denote the closed 3-manifold obtained by performing zero framed surgery on the link $L \subset S^{3}$. A boundary link $L$ in $S^{3}$ is freely slice if $L$ bounds a collection of disjoint, topologically embedded, locally flat discs $D$ in $D^{4}$ such that $\pi_{1}\left(D^{4} \backslash D\right)$ is a free group generated by the meridians of $L$. We recall the following standard proposition.

Proposition 4.1 An m-component boundary link $L$ is freely slice if and only if there is a compact topological 4-manifold $X$ with $\partial X=M_{L}$ such that $\pi_{1}(X)$ is the free group on the $m$ meridians and $H_{2}(X ; \mathbb{Z})=0$.

We remark that such a 4-manifold $X$ is homotopy equivalent to $\bigvee^{m} S^{1}$ by [7, Proposition 11.6C(1)].

Proof of Proposition 4.1 If $L$ is freely slice, the exterior of the slicing discs has the properties required of $X$ (we are using that locally flat embedded discs have normal bundles, by [7, Section 9.3], in order to consider the exterior). For the converse, suppose there is a 4-manifold $X$ satisfying the stated properties. Attach $m$ 2-handles $D^{2} \times D^{2}$ with core $D^{2} \times\{0\}$ to $X$, with attaching circles $\partial D^{2} \times\{0\}$ glued along the meridians of $L$, with zero framings induced from $S^{3}$. The resulting 4-manifold $Y$ is contractible, 
and its boundary $\partial Y$ is homeomorphic to $S^{3}$. By Freedman [9], $Y$ is homeomorphic to $D^{4}$. The belt spheres $\{0\} \times \partial D^{2}$ of the 2-handles are sent to $L \subset S^{3}$ under the homeomorphism $\partial Y \rightarrow S^{3}$, and the cocores $\{0\} \times D^{2}$ of the 2-handles provide slice discs for $L$. Then $X$ is the exterior of the slice discs and $\pi_{1}(X)$ is free, so $L$ is freely slice.

We will prove Theorem A by constructing a topological 4-manifold $X$ satisfying the criteria of Proposition 4.1. In order to find such an $X$, we will need to apply a theorem of Freedman and Quinn from [7, Chapter 6]. Let us introduce the terminology necessary to state this theorem.

A model transverse pair is defined to be two copies of $S^{2} \times D^{2}$ plumbed together once. In other words, a regular neighbourhood of $\left(S^{2} \times\{p\}\right) \cup\left(\{p\} \times S^{2}\right) \cong S^{2} \vee$ $S^{2}$ in $S^{2} \times S^{2}$, where $p \in S^{2}$. Take an arbitrary number of model transverse pairs $N_{1}, \ldots, N_{\ell}$, perform some number of further plumbings, which may or may not be self plumbings, and embed the result into a (topological) 4-manifold $M$. A topological embedding is simply a map that is a homeomorphism onto its image. Call the resulting map $f: \bigsqcup_{i} N_{i} \rightarrow M$ an immersed union of transverse pairs. We say that $f$ has algebraically trivial intersections if the further plumbings introduced by $f$ can be arranged in pairs with Whitney discs immersed in $M$ (the Whitney discs are a priori permitted to intersect the image of the $N_{i}$ ). In addition, we say that $f$ is $\pi_{1}$-null if the inclusion induced map $\pi_{1}\left(f\left(\bigsqcup_{i} N_{i}\right)\right) \rightarrow \pi_{1}(M)$ is trivial for every choice of basepoint in $\bigsqcup_{i} N_{i}$.

A 5-dimensional $s$-cobordism rel $\partial$ is a triple $\left(Z ; \partial_{0} Z, \partial_{1} Z\right)$, where $Z$ is a compact (topological) 5-manifold whose boundary is split into three pieces $\partial Z \cong \partial_{0} Z \cup \partial_{1} Z \cup$ $(P \times[0,1])$ such that $\partial_{i} Z \cap(P \times[0,1])=\partial\left(\partial_{i} Z\right)=P \times\{i\}$ for $i=0,1$, and each inclusion $\partial_{i} Z \hookrightarrow Z$ is a simple homotopy equivalence. We say that $\partial_{0} Z$ and $\partial_{1} Z$ are $s$-cobordant rel $\partial$.

The next theorem says that, under a strong $\pi_{1}$-null hypothesis, we may perform surgery up to s-cobordism.

Theorem 4.2 ([7, Theorem 6.1]) Let $M$ be a compact connected topological 4-manifold with (possibly empty) boundary. Let $f: \bigsqcup_{i} N_{i} \rightarrow M$ be an immersed union of transverse pairs. If $f$ is $\pi_{1}$-null and has algebraically trivial intersections, then $f$ is s-cobordant to an embedding. That is, there is an s-cobordism $\left(Z ; M, M^{\prime}\right)$ rel $\partial$ and a map $F: \bigsqcup_{i} N_{i} \times I \rightarrow Z$ such that $\left.F\right|_{\bigsqcup_{i} N_{i} \times\{0\}}=f$ and $\left.F\right|_{\bigsqcup_{i} N_{i} \times\{1\}}$ is an embedding of $\bigsqcup_{i} N_{i}$ into $M^{\prime}$.

In the next section we will prove the following result, which forms the core of the proof of Theorem A.

Proposition 4.3 Let L be an m-component boundary link with a boundary link Seifert surface admitting a homotopically trivial ${ }^{+}$good basis. Then there exists a compact smooth 4-manifold $W$ with $\partial W=M_{L}, \pi_{1}(W)$ free on the $m$ meridians of $L$, and $\mathrm{H}_{2}(W ; \mathbb{Z}) \cong \mathbb{Z}^{2 g}$ for some $\mathrm{g} \geq 0$. Moreover, there is a basisfor $\mathrm{H}_{2}(W ; \mathbb{Z})$ represented by a $\pi_{1}$-null immersed union of transverse pairs with algebraically trivial intersections.

Assuming the proposition, we give the proof of our main theorem, whose statement we recall for the convenience of the reader. Suppose that $L$ is a good boundary link 
that has a Seifert surface admitting a homotopically trivial ${ }^{+}$good basis. Then $L$ is freely slice.

Proof of Theorem A assuming Proposition 4.3 Apply Theorem 4.2 to $W$, to find a 4manifold $W^{\prime} s$-cobordant rel $\partial$ to $W$, via an $s$-cobordism $\left(Z ; W, W^{\prime}\right)$ with maps

$$
F_{1}, \ldots, F_{2 g}: S^{2} \times[0,1] \rightarrow Z
$$

such that the immersed spheres $\left.F_{i}\right|_{S^{2} \times\{0\}}: S^{2} \rightarrow W$ represent a basis for $H_{2}(W ; \mathbb{Z})$ and $h_{i}:=\left.F_{i}\right|_{S^{2} \times\{1\}}: S^{2} \rightarrow W^{\prime}$ for $i=1, \ldots, 2 g$ are framed embeddings with geometric intersections

$$
\left|h_{i} \pitchfork h_{j}\right|= \begin{cases}1 & \text { if }\{i, j\}=\{2 k-1,2 k\} \text { for some } k \\ 0 & \text { otherwise }\end{cases}
$$

Since $Z$ is an $s$-cobordism, $\left\{h_{i}\left(S^{2}\right)\right\}$ forms a basis of $H_{2}\left(W^{\prime} ; \mathbb{Z}\right)$ as well. Perform surgery on the 2-spheres $h_{2}\left(S^{2}\right), h_{4}\left(S^{2}\right), \ldots, h_{2 g}\left(S^{2}\right)$ to convert $W^{\prime}$ into a compact topological 4-manifold $W^{\prime \prime}$ with $\partial W^{\prime \prime} \cong \partial W^{\prime} \cong \partial W \cong M_{L}$. Since each of the spheres $h_{2 k}\left(S^{2}\right)$ has a geometric dual, the surgeries kill $H_{2}$ and preserve $\pi_{1}$. Thus $\pi_{1}\left(W^{\prime \prime}\right)$ is free on the meridians of $L$ and $H_{2}\left(W^{\prime \prime} ; \mathbb{Z}\right)=0$. By Proposition 4.1 with $X=W^{\prime \prime}, L$ is freely slice.

\section{Construction of the 4-manifold $W$}

Our goal is to construct a 4-manifold $W$ with the properties stated in Proposition 4.3. The construction in this section will be done completely in the smooth category. The description of $W$ given below is closely related to a cobordism used in the proof of [2, Theorem 8.9]. Let $L$ be an $m$-component boundary link with a boundary link Seifert surface $V=\bigsqcup_{k=1}^{m} V_{k}$ that admits a homotopically trivial ${ }^{+}$good basis $\left\{a_{i}, b_{i}\right\}$. Let $g_{k}$ be the genus of $V_{k}$ and let $g=\sum_{k=1}^{m} g_{k}$. Take a bicollar of $V_{k}$ in $S^{3}$ and identify the bicollar with $V_{k} \times[-1,1]$. The bicollar consists of one 3-dimensional 0-handle $D^{0} \times D^{3}$ and $2 g_{k}$ 1-handles $D^{1} \times D^{2}$ corresponding to the curves $a_{i}, b_{i}$, as shown in Fig. 2. For $i$ such that $a_{i} \cup b_{i} \subset V_{k}$, let $\beta_{i}, \gamma_{i}$ and $\delta_{i}$ be the curves in $V_{k} \times[-1,1]$ shown in Fig. 2. The curve $\beta_{i}$ bounds a disc in $V_{k}=V_{k} \times 0$. The $4 g-m$ curves $a_{i}, \beta_{i}, \gamma_{i}$ and $\delta_{i}$, viewed as curves in $S^{3}$ under the bicollar inclusions, form a Kirby diagram. The dotted curves $\beta_{i}$ represent 1 -handles, while $a_{i}, \gamma_{i}$ and $\delta_{i}$ are zero-framed attaching circles for 2-handles. Note that the zero framing of our curves in the standard embedding picture in Fig. 2 corresponds to the zero framing under the bicollar embedding, since the Seifert pairing of $V_{k}$ vanishes on $\left(a_{i}, a_{i}\right)$ and $\left(b_{i}, b_{i}\right)$. Let $W$ be the 4-manifold described by this Kirby diagram.

The boundary of $W$ is diffeomorphic to the zero surgery manifold $M_{L}$, by the arguments of [12, pp. 147-148] and [2, Lemma 8.10]. We describe the proof since we need to keep track of meridians under $M_{L} \cong \partial W$. Regard the Kirby diagram as a surgery presentation of the 3-manifold $\partial W$ consisting of zero framed curves, ignoring the dots on $\beta_{i}$. Slide each $\beta_{i}$ over $a_{i}$ twice, once from the inside of $a_{i}$ and once from 


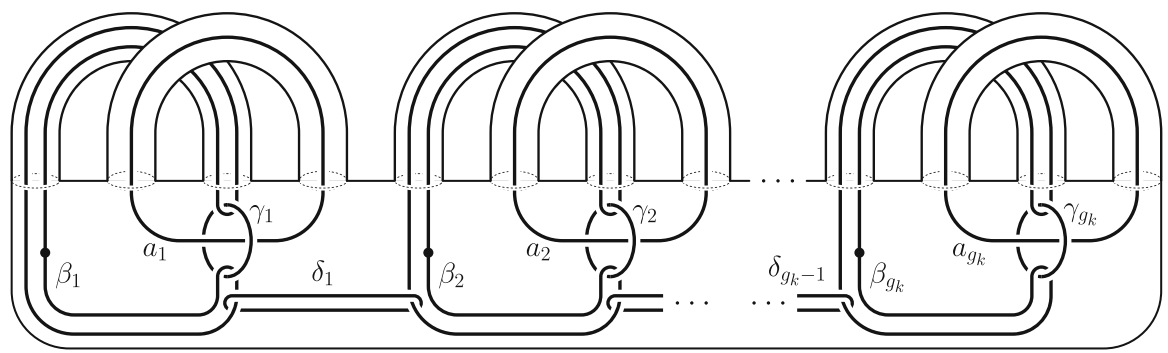

Fig. 2 A Kirby diagram consisting of $a_{i}, \beta_{i}, \gamma_{i}$ and $\delta_{i}$ in the bicollar $V_{k} \times[-1,1]$. For brevity, the indices $i$ such that $a_{i} \cup b_{i} \subset V_{k}$ are assumed to be $1,2, \ldots, g_{k}$. The curves $a_{i}, \gamma_{i}$ and $\delta_{i}$ are all zero framed

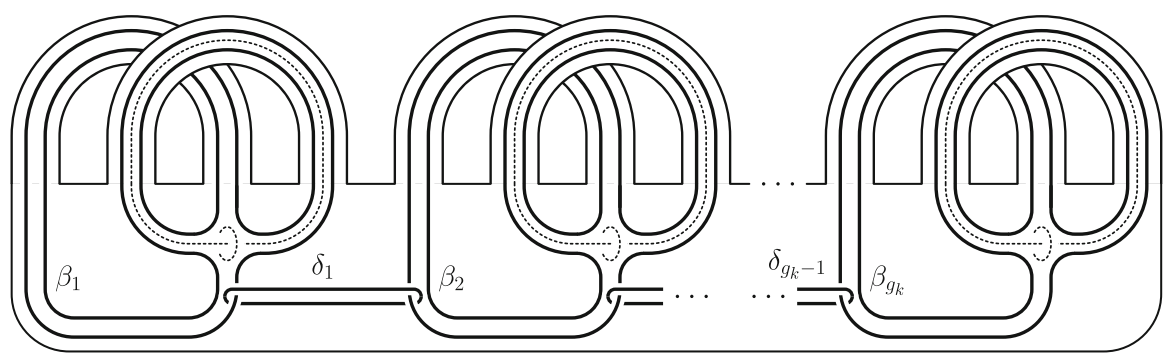

Fig. 3 The surgery curves obtained after handle sliding. The curves $\beta_{i}$ and $\delta_{i}$ are all zero framed

the outside, to obtain the surgery diagram in Fig. 3. We abuse notation and still denote the resulting curves by $\beta_{i}$.

Eliminate, in pairs, the curves $a_{i}$ and $\gamma_{i}$ shown as dashed circles in Fig. 3. Slide the new $\beta_{1}, g_{k}-1$ times, toward the right hand side over the new $\beta_{2}, \ldots, \beta_{g_{k}}$, and finally eliminate $\delta_{i}$ and $\beta_{i+1}$ in pairs for $i=1, \ldots, g_{k}-1$. Now the diagram consists of zero framed curves isotopic to $L$. This shows that $\partial W \cong M_{L}$, as desired. In addition, for $\beta_{i} \subset V_{k}$, a meridian of $\beta_{i}$ is sent to a meridian of the $k$ th component of $L$ under this diffeomorphism.

Claim A The fundamental group $\pi_{1}(W)$ is the free group on the $m$ meridians of $L$.

Proof To compute $\pi_{1}(W)$, begin with $\pi_{1}\left(D^{4} \cup 1\right.$-handles), which is free on the meridians of the curves $\beta_{i}$. The 2-handles attached along $a_{i}$ and $\gamma_{i}$ do not give relators, since $a_{i}$ and $\gamma_{i}$ are null-homotopic in $D^{4} \cup$ (1-handles). This can be seen, for instance, by inspecting their intersections with the disc in $V_{k}$ bounded by $\beta_{i}$. The 2-handles attached along $\delta_{i}$ give relations identifying meridians of all $\beta_{i}$ lying on the same $V_{k}$. So we have $m$ generators, one for each $V_{k}$. We already verified that these generators are meridians of $L$ in $\partial W \cong M_{L}$.

Next, we construct $2 g$ immersed spheres $\Sigma_{1}, \ldots, \Sigma_{2 g}$ in $W$. Apply Lemma 3.3 to $J=\left\{a_{i}, b_{i}^{\prime}\right\}_{i=1}^{g}$ and $K=\left\{b_{i}^{\prime}\right\}_{i=1}^{g}$ to obtain collections of discs $\left\{\Delta_{i}^{+}\right\}_{i=1}^{2 g}$ and $\left\{\Delta_{i}\right\}_{i=1}^{g}$ in $D^{4}$ satisfying 
Fig. 4 The genus one surface $T_{i}^{\circ}$ with $\partial T_{i}^{\circ}=\gamma_{i}$. The push-off $b_{i}^{\prime}$ of $b_{i}$ is either the one shown, or a parallel on the opposite side of $b_{i}$, depending on the normal direction used to take $b_{i}^{\prime}$. The parallel $b_{i}^{\prime+}$ is between $b_{i}$ and $b_{i}^{\prime}$. The dashed $\operatorname{arc} c_{i}$ on $T_{i}^{\circ}$ joins the intersection $a_{i} \cap T_{i}^{\circ}=a_{i} \cap b_{i}$ to the parallel $b_{i}^{\prime+}$, without intersecting $b_{i}^{\prime}$

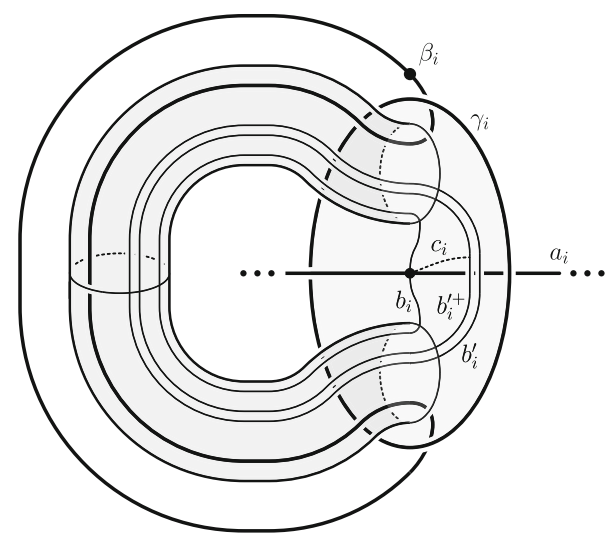

$$
\begin{array}{ll}
\partial \Delta_{i}^{+}=a_{i}, \partial \Delta_{g+i}^{+}=b_{i}^{\prime+}, \partial \Delta_{i}=b_{i}^{\prime} & \text { for } 1 \leq i \leq g, \\
\Delta_{i} \cap \Delta_{j}=\varnothing & \text { for } i \neq j, \text { and } \\
\Delta_{i} \cap \Delta_{j}^{+}=\varnothing & \text { for all } i \text { and } j .
\end{array}
$$

Here $b_{i}^{\prime+}$ is a zero linking parallel of $b_{i}^{\prime}$ as before. For each of these discs, perform interior twists [7, Section 1.3] if necessary to arrange that the signs of the self intersections add up to zero. It follows that the discs induce the zero framing on the boundary. Note that the discs can also be assumed to be smoothly immersed and transverse to each other. We will use the discs to build our immersed spheres.

First, for $i=1, \ldots, g$, cap off the disc $\Delta_{i}^{+}$with the core $D^{2} \times\{0\}$ of the 2-handle $D^{2} \times D^{2}$ attached along $a_{i}=\partial \Delta_{i}^{+}$. Let

$$
\Sigma_{2 i-1}:=\left(D^{2} \times\{0\}\right) \underset{a_{i}}{\cup} \Delta_{i}^{+} \quad(i=1, \ldots, g)
$$

be the resulting immersed spheres. They are half of our spheres. Since the 2-handle is attached along the zero framing, the normal bundle of $\Sigma_{2 i-1}$ is trivial, so $\Sigma_{2 i-1}$ is framed.

Now we define the spheres $\Sigma_{2 i}$, for $i=1, \ldots, g$. Take the genus one surface $T_{i}^{\circ} \subset W$ shown in Fig. 4. It is bounded by $\gamma_{i}$ and contains $b_{i}$. Cap off $T_{i}^{\circ}$ with the core of the 2-handle attached along $\gamma_{i}$, to obtain a torus in $W$, which we call $T_{i}$.

Note that the normal direction of $b_{i}$ in $T_{i}^{\circ}$ agrees with a normal direction of the Seifert surface. So we may assume that $b_{i}^{\prime}$ is a parallel of $b_{i}$ on $T_{i}^{\circ}$, as shown in Fig. 4. Also, since the Seifert pairing vanishes on $\left(b_{i}, b_{i}\right)$, we may assume that the zero linking parallel $b_{i}^{\prime+}$ is on $T_{i}^{\circ}$ too. In addition, we assume that $b_{i}^{\prime+}$ is a translate of $b_{i}^{\prime}$ taken along the normal direction of $b_{i}^{\prime}$ toward $b_{i}$, as shown in Fig. 4. That is, $b_{i}^{\prime+}$ lies between $b_{i}^{\prime}$ and $b_{i}$ on $T_{i}^{\circ}$. (This will be crucial in verifying $\pi_{1}$-nullity later.) Contract $T_{i}$ using the discs $\Delta_{i}$ and $\Delta_{g+i}^{+}$to obtain a 2-sphere $\Sigma_{2 i}$. More precisely, take the annulus cobounded by $b_{i}^{\prime}$ and $b_{i}^{\prime+}$ in $T_{i}^{\circ}$, and remove it from $T_{i}$ to get the complementary annulus $A_{i}$. Attach the discs $\Delta_{i}$ and $\Delta_{g+i}^{+}$to $A_{i}$ along the boundary, to construct our 2-spheres 
Fig. 5 A schematic diagram of construction of $\Sigma_{2 i-1}$ and $\Sigma_{2 i}$. The dot on the circle $a_{i}$ represents the intersection $a_{i} \cap A_{i}$ shown in Fig. 4. Other dots represent potentially existing intersections

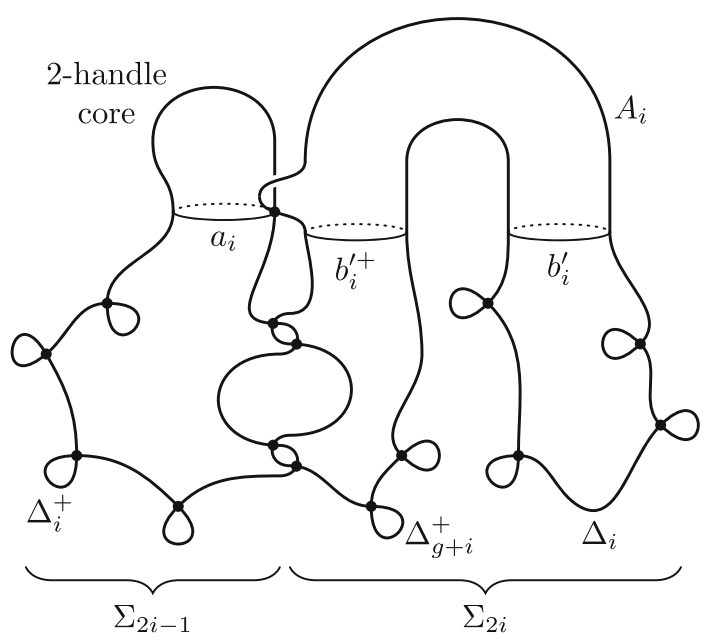

$$
\Sigma_{2 i}:=\Delta_{i} \underset{b_{i}^{\prime}}{\cup} A_{i} \underset{b_{i}^{\prime+}}{\cup} \Delta_{g+i}^{+} \quad(i=1, \ldots, g) .
$$

Since $\Delta_{i}, \Delta_{g+i}^{+}$and $A_{i}$ induce the zero framing on $b_{i}^{\prime}$ and $b_{i}^{\prime+}, \Sigma_{2 i}$ is framed.

Claim B The homology $H_{2}(W ; \mathbb{Z})$ is the free abelian group of rank $2 g$ generated by the classes of $\Sigma_{i}$.

Proof Consider the cellular chain complex associated to the handle decomposition of $W$. In particular we have $C_{2}(W ; \mathbb{Z})=H_{2}\left(W, W^{(1)} ; \mathbb{Z}\right)$ where $W^{(1)}=D^{4} \cup$ 1-handles. Inspect the classes of 2-handle attaching curves in $H_{1}\left(W^{(1)} ; \mathbb{Z}\right)$, in the $\pi_{1}$ computation above, to verify that $H_{2}(W ; \mathbb{Z}) \cong \mathbb{Z}^{2 g}$ and that the 2-handle cores bounded by $a_{i}$ and $\gamma_{i}$ are generators. Note that each $\Sigma_{i}$ uses exactly one of these 2-handle cores, and that other building blocks $\Delta_{i}, \Delta_{i}^{+}$and $T_{i}^{\circ}$ lie in $W^{(1)}$. It follows that $\Sigma_{i}$ equals the corresponding 2-handle core as a 2-cycle in the cellular chain complex $C_{2}(W ; \mathbb{Z})=H_{2}\left(W, W^{(1)} ; \mathbb{Z}\right)$.

Note that the framed spheres $\Sigma_{2 i-1}$ and $\Sigma_{2 i}$ have a distinguished transverse intersection at $a_{i} \cap T_{i}=a_{i} \cap b_{i}$. This is also depicted in Fig. 4. So, (a regular neighborhood of) $\Sigma_{2 i-1} \cup \Sigma_{2 i}$ can be viewed as an immersed pair of transverse spheres.

Claim $C$ The union $\bigcup_{i=1}^{2 g} \Sigma_{i}$ of the $g$ pairs has algebraically trivial intersections.

Proof First, we investigate the intersections of the discs $\Delta_{i}$ and $\Delta_{i}^{+}$. Recall that the signs of the self intersections of each disc add up to zero, that is, the $\mathbb{Z}$-valued self intersection number vanishes. Since the discs lie in $D^{4}$, which is simply connected, it follows that all the self intersections can be paired up by (possibly immersed) Whitney discs. For $\Delta_{i}^{+}$and $\Delta_{j}^{+}$with $i \neq j$, their $\mathbb{Z}$-valued intersection number vanishes because the linking number of $\partial \Delta_{i}^{+}$and $\partial \Delta_{j}^{+}$is zero. So, their intersections can also be paired up by Whitney discs in $D^{4}$. Moreover, recall that $\Delta_{i} \cap \Delta_{j}=\emptyset$ for $i \neq j$ and $\Delta_{i}^{+} \cap \Delta_{j}=\varnothing$ for all $i$ and $j$. 
Now, consider the spheres $\Sigma_{i}$. Since they are built using $\Delta_{i}, \Delta_{i}^{+}, A_{i}$ and 2-handle cores, the (self and non-self) intersections of the spheres, with the exception of the distinguished transverse intersection of the dual pair $\left(\Sigma_{2 i-1}, \Sigma_{2 i}\right)$ at $a_{i} \cap T_{i}=a_{i} \cap b_{i}$, are exactly the (self and non-self) intersections of the discs $\Delta_{i}$ and $\Delta_{i}^{+}$. These can all be paired up by immersed Whitney discs by the preceding paragraph. This proves the claim.

Claim D The union $\bigcup_{i=1}^{2 g} \Sigma_{i}$ is $\pi_{1}$-null in $W$.

Proof Recall that $\Sigma_{2 i-1}$ is the union of $\Delta_{i}^{+}$and a 2-handle core disc. Remove these core discs from $\bigcup_{i=1}^{2 g} \Sigma_{i}$ to get a 2-complex $Y$. That is, $Y=\bigcup_{i=1}^{g}\left(\Delta_{i}^{+} \cup \Sigma_{2 i}\right)$. Since the inclusion induces an isomorphism $\pi_{1}(Y) \cong \pi_{1}\left(\bigcup_{i} \Sigma_{i}\right)$ for any basepoint, $\bigcup_{i=1}^{2 g} \Sigma_{i}$ is $\pi_{1}$-null if and only if $Y$ is $\pi_{1}$-null in $W$. Remove from $Y$ the discs $\Delta_{i}$ used to construct $\Sigma_{2 i}$, for all $i$, to obtain a 2-complex $Y_{0}$ given by

$$
Y_{0}:=\bigcup_{i=1}^{g}\left(\Delta_{i}^{+} \cup\left(A_{i} \underset{b_{i}^{\prime+}}{\cup} \Delta_{g+i}^{+}\right)\right) .
$$

Since (int $\left.\Delta_{i}\right) \cap Y_{0}=\emptyset$ and $\Delta_{i} \cap \Delta_{j}=\emptyset$ for $i \neq j, \pi_{1}(Y)$ is the free product of $\pi_{1}\left(Y_{0}\right)$ and the groups $\pi_{1}\left(\Delta_{i}\right)$, by the Seifert-van Kampen theorem. Each $\Delta_{i}$ is $\pi_{1}$-null since $\Delta_{i}$ is contained in $D^{4}$. Therefore, $Y$ is $\pi_{1}$-null if and only if $Y_{0}$ is $\pi_{1}$-null.

Now, consider the arc $c_{i}$ on $A_{i}$ shown in Fig. 4, which joins the point $a_{i} \cap A_{i}$ to the boundary circle $b_{i}^{\prime+}$. From Fig. 4, we see that the annulus $A_{i}$ strongly deformation retracts onto the 1 -subcomplex $c_{i} \cup b_{i}^{\prime+}$. It follows that $Y_{0}$ strongly deformation retracts onto

$$
Y_{1}=\bigcup_{i=1}^{g}\left(\Delta_{2 i-1}^{+} \cup c_{i} \cup \Delta_{2 i}^{+}\right) .
$$

Observe that $c_{i}$ is contained in $W^{(1)}=D^{4} \cup 1$-handles. Moreover, $c_{i}$ is disjoint from the 1-handles of $W$, since $c_{i}$ does not intersect the obvious 2-disc in $V_{k}$ bounded by the dotted circle $\beta_{i}$, as seen in Fig. 4 (see also Fig. 2). Here, it is crucial that the parallel $b_{i}^{\prime+}$ lies between $b_{i}$ and $b_{i}^{\prime}$; if $b_{i}^{\prime+}$ were on the opposite side of $b_{i}^{\prime}$, then $c_{i}$ would meet (the cocore of) the 1-handle corresponding to $\beta_{i}$. It follows that the 2-complex $Y_{1}$ is contained in $D^{4}$. Therefore $Y_{1}$ is $\pi_{1}$-null, and consequently $Y_{0}, Y$ and $\bigcup_{i=1}^{2 g} \Sigma_{i}$ are all $\pi_{1}$-null. This proves the claim, and completes the proof of Proposition 4.3.

Remark 5.1 In the above $\pi_{1}$-nullity proof, note that there might have been a homotopically essential loop in $\Sigma_{2 i-1} \cup \Sigma_{2 i}$, passing through the distinguished transverse intersection $a_{i} \cap A_{i}$ exactly once, from one sphere to another: start from $a_{i} \cap A_{i}$, depart into $\Delta_{i}^{+} \subset \Sigma_{2 i-1}$ and proceed to reach an intersection with $\Delta_{g+i}^{+} \subset \Sigma_{2 i}$, and then go back to the point $a_{i} \cap A_{i}$ through $\Sigma_{2 i}$. See Fig. 5. This occurs since the discs $\Delta_{i}^{+}$and $\Delta_{g+i}^{+}$used to construct the dual 2-spheres $\Sigma_{2 i-1}$ and $\Sigma_{2 i}$ are allowed to meet. Our subtle choice of $b_{i}^{\prime+}$ from the two possibilities, together with the use of the arc $c_{i}$, is crucial in showing that such a loop is null-homotopic in $W$. 
This subtlety is peculiar to our case, compared with the proof of the earlier result of Freedman and Teichner [13] that a Whitehead double of a homotopically trivial ${ }^{+}$ link is freely slice. In their construction, immersed discs used to construct a pair of dual 2-spheres are always disjoint, and thus their verification of the $\pi_{1}$-nullity does not involve this complication of our case.

Remark 5.2 It can also be shown that $\pi_{2}(W)=H_{2}\left(W ; \mathbb{Z}\left[\pi_{1}(W)\right]\right)$ is the free module of rank $2 g$ over the group ring $\mathbb{Z}\left[\pi_{1}(W)\right]$ generated by our spheres $\Sigma_{i}$. Since we do not use this, we omit the details.

\section{Comparison with previously known results}

Here is a summary of previously known results on freely slicing good boundary links. Recall that any Whitehead double of a pairwise linking number zero link is a good boundary link. For statement (4) below, we need some terminology from [12]. A link is said to be a $\partial^{2}$-link if it bounds a boundary link Seifert surface $V$ with basis $\left\{\alpha_{i}, \beta_{i}\right\}$ for $H_{1}(V ; \mathbb{Z})$, for which the Seifert matrix is the diagonal sum of matrices of the form $\left[\begin{array}{rr}0 & \varepsilon \\ 1-\varepsilon & 0\end{array}\right]$ with $\varepsilon=0$ or 1 , and the homology classes $\beta_{i} \in H_{1}(V ; \mathbb{Z})$ can be realised by a collection of disjoint simple closed curves $b_{i}$ on $V$ that when considered as a link in its own right is itself a boundary link $J$. Moreover we require that $J$ bounds a boundary link Seifert surface in $S^{3}$ whose interior is disjoint from $V$.

(1) Freedman [8] showed that a knot is freely slice if and only if it has Alexander polynomial one, that is if it is a good boundary link.

(2) Freedman [10] showed that Whitehead doubles of boundary links are freely slice good boundary links.

(3) Freedman [11] showed that a Whitehead double of a 2-component link $L$ is freely slice if and only if $L$ has linking number zero.

(4) Freedman [12] showed that a $\partial^{2}$-link $L$ is freely slice.

(5) Freedman and Teichner [13] showed that a Whitehead double of a homotopically trivial $^{+}$link is freely slice.

In addition to the above list of results on freely slice boundary links, CochranFriedl-Teichner [1] produced new slice links from a satellite construction, whilst the first and third named authors [3, Theorem F] showed that any iterated ramified 4-fold Whitehead double is slice. Moreover, in [3], they also introduced distorted Whitehead doubles, and showed that they are slice too. Neither the results of [1] nor [3] address whether the slice discs are free.

Our result does not recover statement (1). Instead, for an Alexander polynomial one knot $K$, form the 4-manifold $W_{0}$ as in our proof. Then the generators of $\pi_{2}\left(W_{0}\right)$ that we construct need not be $\pi_{1}$-null. But, since $\pi_{1}\left(W_{0}\right)$ is the good group $\mathbb{Z}$, it turns out that one can use ordinary topological surgery to produce a slice disc exterior for $K$, instead of surgery up to $s$-cobordism. This is essentially the argument of [7, Theorem 11.7B].

In what follows, we discuss the relationship of our theorem with the work (2)-(5). Also, with reference to (2)-(5), in Sect. 6.1 we will provide examples of links that are freely slice by our Theorem A, but which are neither Whitehead doubles nor $\partial^{2}$-links. 
Lemma 6.1 Let $J$ be a link with trivial pairwise linking numbers. Then the standard Seifert surface for the boundary link $\mathrm{Wh}(J)$ consisting of genus one surfaces has a good basis which is homotopically trivial ${ }^{+}$if and only if $J$ is a homotopically trivial ${ }^{+}$ link.

Proof Let $J_{i}$ be the $i$ th component of $J$. Figure 1 shows the standard genus one Seifert surface for the Whitehead double of $J_{i}$. Let $a_{i}$ and $b_{i}$ be the curves on the Seifert surface shown in Fig. 1. Since $J$ has trivial linking numbers, the Seifert matrix for the boundary link $\mathrm{Wh}(J)$ with respect to $\left\{a_{i}, b_{i}\right\}$ is the diagonal sum of $2 \times 2$ matrices of the form $\left[\begin{array}{cc}0 & \varepsilon \\ 1-\varepsilon & 0\end{array}\right]$ with $\varepsilon=0$ or 1 . It follows that $\left\{a_{i}, b_{i}\right\}$ is a good basis.

As in the definition of a homotopically trivial ${ }^{+}$good basis, let $b_{i}^{\prime}$ be a translate of $b_{i}$ along a normal direction to the Seifert surface such that $\operatorname{lk}\left(a_{i}, b_{i}^{\prime}\right)=0$, and let $K:=\bigsqcup b_{i}^{\prime}$. Observe that $a_{i}, b_{i}$ and $b_{i}^{\prime}$ are zero linking parallels of $J_{i}$. Thus both $K \cup a_{i}$ and $K \cup b_{i}$ are isotopic to $J \cup J_{i}^{+}$. It follows that the link $J$ is homotopically trivial ${ }^{+}$ if and only if the good basis $\left\{a_{i}, b_{i}\right\}$ is homotopically trivial ${ }^{+}$.

Thus (5) is a corollary of Theorem A, as mentioned in the introduction.

A link $L$ is homotopically trivial ${ }^{+}$if and only if all of Milnor's invariants with at most one repeated index are trivial [13, Lemma 2.7]. In particular, boundary links are homotopically trivial $^{+}$since all their Milnor's invariants vanish. Also, a 2-component link with trivial linking number is homotopically trivial ${ }^{+}$, since $\bar{\mu}(112)=\bar{\mu}(122)=0$ for any such link, due to cyclic symmetry and the shuffle relation [20, Theorem 6]. It follows that (5) implies (2) and (3). Therefore (2) and (3) are corollaries of Theorem A too.

By the following lemma, Theorem A implies (4).

Lemma 6.2 A $\partial^{2}$-link has a boundary link Seifert surface admitting homotopically trivial $^{+}$good basis.

Proof Suppose that $L$ is a $\partial^{2}$-link. Choose a boundary link Seifert surface $V$ and disjoint simple closed curves $b_{i}$ on $V$ with the properties described in the definition of a $\partial^{2}$-link. The link $\bigsqcup b_{i}$ bounds a boundary link Seifert surface $W$ that is transverse to $V$ and satisfies $V \cap W=\bigsqcup b_{i}$. Choose disjoint simple curves $a_{i}$ on $V$ that are geometrically dual to the $b_{i}$. That is, the geometric intersections are given by $a_{i} \cdot b_{j}=$ $\delta_{i j}$. Here, we allow that the homology class of $a_{i}$ may be different from the $\alpha_{i}$ in $H_{1}(V ; \mathbb{Z})$ given in the definition of a $\partial^{2}$-link. Let $Y$ be the surface $W$ with an open collar of $\partial W$ removed. Then $\partial Y$ consists of push-offs $b_{i}^{\prime}$ of the $b_{i}$, and $Y$ is a boundary link Seifert surface for the link $K=\bigsqcup b_{i}^{\prime}$.

We claim, in general, that if $K$ is a boundary link with boundary link Seifert surface $Y$ and $\gamma$ is a knot disjoint from $Y$, then $K \cup \gamma$ is homotopically trivial. Applying this to our case, it follows that $K \cup b_{i}$ and $K \cup a_{i}$ are homotopically trivial for each $i$. This shows that $\left\{a_{i}, b_{i}\right\}$ is a homotopically trivial ${ }^{+}$good basis for $L$.

To prove the claim, let $\pi=\pi_{1}\left(S^{3} \backslash K\right)$, and consider the epimorphism $\phi: \pi \rightarrow F$ onto the free group $F$ given by the Pontryagin-Thom construction for $Y$. Since $\gamma$ is disjoint from $Y, \gamma$ lies in the kernel of $\phi$. Since $\phi$ induces an isomorphism $\pi / \pi_{k} \cong$ $F / F_{k}$ between the lower central series quotients for every $k$ by Stallings' theorem [23], $\gamma$ is trivial in $\pi / \pi_{k}$. It follows that $\bar{\mu}_{K \cup \gamma}\left(j_{1} \cdots j_{k}\right)=0$ whenever $j_{1}, \ldots, j_{k-1}$ 
Fig. 6 A string link $\beta$ whose closure is the Whitehead link
Fig. 7 The link $L(\beta)$. The boxes indicate the relevant string links arising as cables of $\beta$
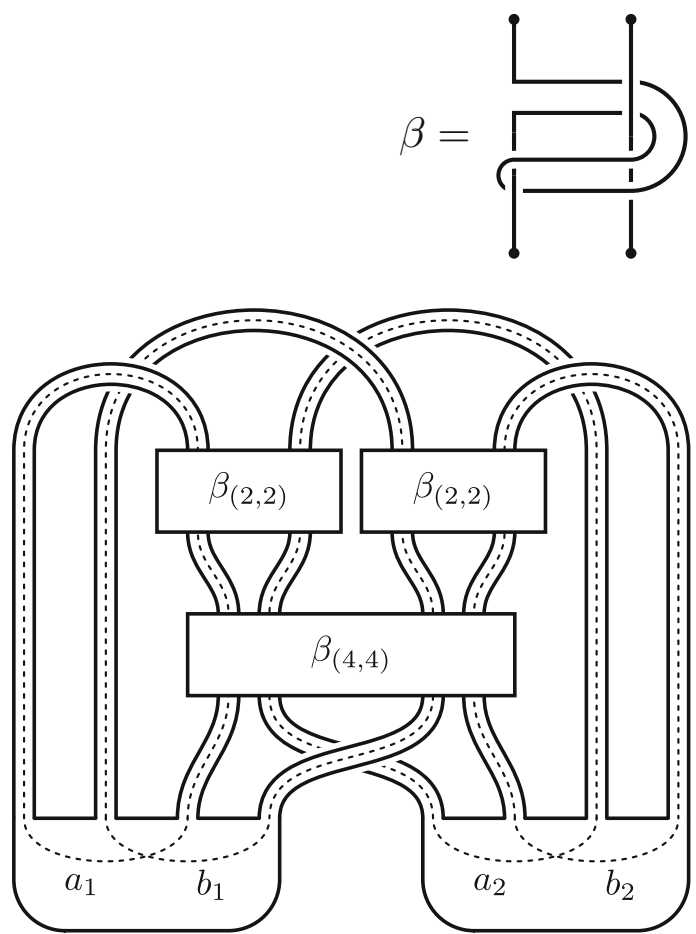

correspond to components of $K$ and $j_{k}$ corresponds to the component $\gamma$. Also, if all of $j_{1}, \ldots, j_{k}$ correspond to components of $K$, then $\bar{\mu}_{K \cup \gamma}\left(j_{1} \cdots j_{k}\right)=\bar{\mu}_{K}\left(j_{1} \cdots j_{k}\right)=0$ since $K$ is a boundary link. The vanishing of these Milnor's invariants, together with cyclic symmetry, in particular implies the vanishing of Milnor's invariants of $K \cup \gamma$ for all non-repeating multi-indices. Thus $K \cup \gamma$ is homotopically trivial [19]. This completes the proof of the claim and therefore of the lemma.

\subsection{Examples that are not Whitehead doubles}

In this section we present the promised examples of links that can be freely sliced using Theorem A, but which are neither Whitehead doubles nor $\partial^{2}$-links.

Let $\beta$ be a 2 -component string link whose closure $\widehat{\beta}$ is a non-slice link with trivial linking number. For instance, $\beta$ could be the string link in Fig. 6, whose closure is the Whitehead link. Let $\beta_{(k, \ell)}$ be the $(k+\ell)$-component string link obtained by replacing the first and second strand of $\beta$ with their untwisted $k$ and $\ell$ cables respectively. Let $L(\beta)$ be the 2-component link shown in Fig. 7.

\section{Corollary 6.3 The link $L(\beta)$ is freely slice if $\widehat{\beta}$ has trivial linking number.}

Proof Recall that a 2-component link with trivial linking number is homotopically trivial $^{+}$. (See the discussion between Lemmas 6.1 and 6.2.) So, in our case, $\widehat{\beta_{(1,2)}}$ and $\widehat{\beta_{(2,1)}}$ are homotopically trivial. 
Figure 7 is drawn in such a way that the Seifert surface ought to be obvious. Consider the good basis $\left\{a_{i}, b_{i}\right\}$ shown, and let $K=b_{1}^{\prime} \cup b_{2}^{\prime}$, where $b_{i}^{\prime}$ is a transport of $b_{i}$ along a normal direction of the surface such that $\operatorname{lk}\left(a_{i}, b_{i}^{\prime}\right)=0$. Then $K=\widehat{\beta}$. Thus $K \cup b_{1}=\widehat{\beta_{(2,1)}}$ and $K \cup b_{2}=\widehat{\beta_{(1,2)}}$ are homotopically trivial. For $i=1,2$, the link $K \cup a_{i}$ is the closure of the product of two 3-component string links $1_{1} \otimes \beta$ and $\beta_{(1,2)}$, where $1_{1} \otimes \beta$ denotes the split union of a 1-component trivial string link and $\beta$. Since $\widehat{\beta_{(1,2)}}$ and $\widehat{\beta}$ are homotopically trivial, it follows that $K \cup a_{i}$ is homotopically trivial. Therefore the good basis $\left\{a_{i}, b_{i}\right\}$ is homotopically trivial ${ }^{+}$. Thus by Theorem $\mathrm{A}$, the link $L(\beta)$ is freely slice.

When $\beta$ is not slice, we cannot see any other way to show that $L(\beta)$ is slice. As an explicit example, let $\beta$ be the Whitehead string link in Fig. 6. Attempts to produce a slicing disc by cutting 1-handles of the given Seifert surface fail. Both components of the link $L(\beta)$ in Fig. 7 are hyperbolic knots (the same hyperbolic knot with volume 13.16319, in fact), as we verified using SnapPy. Note that by contrast the components of a Whitehead double are non-hyperbolic knots. Thus our link does not arise as a Whitehead double. Also $L(\beta)$ bounds a collection of Seifert surfaces whose metabolisers are realised by non-boundary links. In fact each possible metaboliser $\left(a_{1}, a_{2}\right),\left(a_{1}, b_{2}\right),\left(b_{1}, a_{2}\right)$ or $\left(b_{1}, b_{2}\right)$ is a Whitehead link. So $L(\beta)$ does not seem to be a $\partial^{2}$-link. Nevertheless our Theorem A applies to prove that $L(\beta)$ is slice, as shown in Corollary 6.3 .

\section{Questions}

Here are some potentially interesting questions raised by the investigation in this article.

(1) Is there a link that is not homotopically trivial ${ }^{+}$in the sense of Freedman and Teichner, but whose Whitehead double admits a homotopically trivial ${ }^{+}$good basis?

(2) Does every good boundary link have a homotopically trivial ${ }^{+}$good basis?

Put differently, (1) asks whether Theorem A can be used to slice Whitehead doubles to which the Freedman-Teichner result does not apply. If (1) has an affirmative answer, the desired homotopically trivial ${ }^{+}$good basis will be on a non-standard Seifert surface for the Whitehead double. One might ask a generalised question: for two good bases for the same good boundary link, possibly on different Seifert surfaces, is one homotopically trivial ${ }^{+}$if and only if so is the other? This has a negative answer, since one can stabilise a Seifert surface by a genus three Seifert surface for the unknot, and apply the technique of [21]. So one should perhaps refine this version, for example by restricting to the case that all connected components of the Seifert surfaces have genus one.

If (2) has an affirmative answer, then all good boundary links will be freely slice by Theorem A, and consequently topological surgery would work in dimension 4 for arbitrary fundamental groups. A Whitehead double of the Borromean rings might provide a counterexample to (2). 
Acknowledgements JCC was partly supported by National Research Foundation of Korea (NRF) Grant No. 2019R1A3B2067839. Part of this work was done when JCC was visiting the Max Planck Institute for Mathematics in Bonn. MHK thanks Durham University and Anthony Conway for their hospitality during visits to Durham. MHK was partly supported by the POSCO TJ Park Science Fellowship.

Open Access This article is distributed under the terms of the Creative Commons Attribution 4.0 International License (http://creativecommons.org/licenses/by/4.0/), which permits unrestricted use, distribution, and reproduction in any medium, provided you give appropriate credit to the original author(s) and the source, provide a link to the Creative Commons license, and indicate if changes were made.

\section{References}

1. Cochran, T.D., Friedl, S., Teichner, P.: New constructions of slice links. Comment. Math. Helv. 84(3), 617-638 (2009)

2. Cochran, T.D., Orr, K.E., Teichner, P.: Knot concordance, Whitney towers and $L^{2}$-signatures. Ann. Math. (2) 157(2), 433-519 (2003)

3. Cha, J.C., Powell, M.: Casson towers and slice links. Invent. Math. 205(2), 413-457 (2016)

4. Farber, M.S.: Hermitian forms on link modules. Comment. Math. Helv. 66(2), 189-236 (1991)

5. Freedman, M.H., Krushkal, V.: Engel relations in 4-manifold topology. Forum Math. Sigma 4, e22, 57 (2016)

6. Farb, B., Margalit, D.: A Primer on Mapping Class Groups, Princeton Mathematical Series, vol. 49. Princeton University Press, Princeton (2012)

7. Freedman, M.H., Quinn, F.: Topology of 4-Manifolds, Princeton Mathematical Series, vol. 39. Princeton University Press, Princeton (1990)

8. Freedman, M.H.: A surgery sequence in dimension four; the relations with knot concordance. Invent. Math. 68(2), 195-226 (1982)

9. Freedman, M.H.: The topology of four-dimensional manifolds. J. Differ. Geom. 17(3), 357-453 (1982)

10. Freedman, M.H.: A new technique for the link slice problem. Invent. Math. 80(3), 453-465 (1985)

11. Freedman, M.H.: Whitehead 3 is a "slice" link. Invent. Math. 94(1), 175-182 (1988)

12. Freedman, M.H.: Link compositions and the topological slice problem. Topology 32(1), 145-156 (1993)

13. Freedman, M.H., Teichner, P.: 4-manifold topology. II. Dwyer's filtration and surgery kernels. Invent. Math. 122(3), 531-557 (1995)

14. Hillman, J: Algebraic invariants of links, Series on Knots and Everything, 2nd edn., vol. 52. World Scientific Publishing Co. Pte. Ltd., Hackensack (2012)

15. Ko, K.H.: Seifert matrices and boundary link cobordisms. Trans. Am. Math. Soc. 299(2), 657-681 (1987)

16. Krushkal, V.: "Slicing" the Hopf link. Geom. Topol. 19(3), 1657-1683 (2015)

17. Levine, J.P.: Polynomial invariants of knots of codimension two. Ann. Math. (2) 84, 537-554 (1966)

18. Liang, C.C.: An algebraic classification of some links of codimension two. Proc. Am. Math. Soc. 67(1), 147-151 (1977)

19. Milnor, J.W.: Link groups. Ann. Math. (2) 59, 177-195 (1954)

20. Milnor, J.W.: Isotopy of links. Algebraic geometry and topology, a symposium in honor of S. Lefschetz, pp. 280-306. Princeton University Press, Princeton (1957)

21. Park, J.H.: Milnor's triple linking number and derivatives of genus three knots. arXiv:1603.09163 (2016)

22. Sheiham, D.: Invariants of boundary link cobordism. II. The Blanchfield-Duval form, Non-commutative localization in algebra and topology, London Math. Soc. Lecture Note Ser., vol. 330, pp. 143-219. Cambridge Univ. Press, Cambridge (2006)

23. Stallings, J.: Homology and central series of groups. J. Algebra 2, 170-181 (1965)

Publisher's Note Springer Nature remains neutral with regard to jurisdictional claims in published maps and institutional affiliations. 